Aims. The aim of this study was to identify pre-discharge risk factors associated with early inpatient readmission in general adult service users, with a particular focus on modifiable factors. We hypothesised that stability prior to discharge would reduce readmission to inpatient services within 90 days.

Background. Early readmission to inpatient psychiatric services is a poor outcome for service users, staff and the healthcare system. A variety of clinical, demographic and system factors, mostly nonmodifiable, have been investigated previously. The identification of pre-discharge and particularly modifiable factors associated with readmission would give an opportunity for intervention and changes in policy.

Method. 272 medical records of all admissions within an 8 month period to a NHS inner city psychiatric inpatient service were reviewed to identify factors associated with readmission within 90 days of discharge. The data were analysed by simple comparison, calculation of odds ratios and logistic regression.

Result. $26 \%$ of service users were readmitted to the mental health trust within 90 days of discharge. Incidents $(\mathrm{OR}=3.86$; $95 \% \mathrm{CI}$ 1.39-10.75) and psychotropic medication change in the week before discharge $(\mathrm{OR}=2.94 ; 95 \%$ CI $1.43-6.03)$ were significantly associated with readmission, as were the number of previous admissions, and comorbid substance misuse. Successful overnight leave was found to be significantly protective against readmission $(\mathrm{OR}=0.29 ; 95 \%$ CI $0.11-0.72)$.

Conclusion. The ability to predict those at high risk for readmission means they can be targeted for interventions and it can also help develop best practice around inpatient care and the discharge process. The novel findings in this study of pre-discharge modifiable risk factors such as stability and successful overnight leave could have significant implications in discharge planning policy.

\section{Anticonvulsants and antipsychotics for treating anxiety and depressive symptoms in people with alcohol dependence: a systematic review}

Ka Yee Chow*, Katy Jones and Musa Sami

University of Nottingham

${ }^{\star}$ Corresponding author.

doi: 10.1192/bjo.2021.801

Aims. Anxiety and depressive symptoms are frequent in people with alcohol use disorders (AUDs) (approximately 55\%) and are associated with worse outcomes. Current interventions to treat mood symptoms in alcohol-dependent adults, including psychosocial therapies, anxiolytics and antidepressants have shown limited benefits to date. The efficacy of anticonvulsants and antipsychotics have been studied but never previously systematically reviewed. We aimed to assess the efficacy of anticonvulsants and antipsychotics in treating anxiety and depressive symptoms in alcohol-dependent adults.

Method. A literature search of MEDLINE, EMBASE and PsycINFO was performed through October 2020 to identify all English-language articles of double-blinded randomised controlled trials that included adults with AUDs who were treated with an anticonvulsant or antipsychotic for at least four weeks. A combination of search terms was used to describe the AUDs, study medications and the primary outcome. Participants with other psychiatric disorders were excluded. Mean changes in anxiety and depressive scores were evaluated in addition to the adverse events and withdrawal rates. The risk of bias of each study was also assessed.

Result. Of 393 citations identified, 23 studies (2823 participants) met the inclusion criteria. Eighteen studies examined ten different anticonvulsants, while five studies examined two antipsychotics.
The heterogeneity between the trials' methodology led to conflicting results; however, as the low-quality trials were excluded, the majority agreed that anticonvulsants and antipsychotics were not superior in moderating anxiety and depressive symptoms of alcohol-dependent adults. All antipsychotics were safe and well-tolerated, but adverse events were associated with several anticonvulsants (high dose baclofen, gabapentin, topiramate and valproate).

Conclusion. Current information on anticonvulsants and antipsychotics is insufficient to extrapolate the benefits of treating anxiety and depressive symptoms in alcohol-dependent adults. Further research must be conducted into improving the quality of reporting and understanding the comorbidity's underlying mechanism, and alternative treatment approaches. Attitudes of medical students towards psychiatry and
mental illnesses: a cross-sectional study from Pakistan

Mishal Fatima ${ }^{3}$, Raja Adnan Ahmed², Muhammad Hamza ${ }^{1}$, Syed Muhammad Jawad Zaidi ${ }^{1 \star}$, Hassan Nadeem ${ }^{4}$ and Mehwish Kaneez ${ }^{1}$

${ }^{1}$ Rawalpindi Medical University; ${ }^{2}$ Consultant Psychiatrist, Cmw Taf Morhannwg University Health board; ${ }^{3}$ Final Year MBBS student, Rawalpindi Medical University and ${ }^{4}$ Jinnah Sindh Medical Univeristy, Dudley group NHS Foundation Trust ${ }^{\star}$ Corresponding author.

\section{doi: 10.1192/bjo.2021.802}

Aims. The increasing burden of mental disorders coupled with the social stigmatization in Pakistan is an immense barrier in combating the emerging mental health crisis. The low number of qualified psychiatrists and poor intake in post-graduate psychiatry training programs in the region further complicates the problem. Thus, our study aims to assess the attitudes of Pakistani medical students towards psychiatry. Furthermore, we also aim to evaluate how experience and different levels of exposure to psychiatry among students affect their attitudes towards psychiatry as a career choice. Method. This cross-sectional study was conducted via an online survey made on Google Forms. A total of 831 medical students studying across various private and public medical institutions of Pakistan responded to the survey. The questionnaire comprised of demographical details (gender, age, institution, and academic year) exposure to psychiatry, duration of psychiatry rotation, and personal experience with mental illness. The attitudes of medical students towards psychiatry were evaluated using the English version of the 30-item Attitudes Towards Psychiatry (ATP-30) scale. Chi-square test and multiple regression with backward method were used to analyze the data.

Result. The Cronbach's alpha value of the ATP-30 scale was 0.830 . The participants in our study had a mean score of 107.6 \pm 12 on ATP-30. Overall, most participants had a positive attitude towards psychiatry. Multiple regression analysis revealed a significant model pertaining to predictors of attitude toward psychiatry $(\mathrm{F}(\mathrm{df})=11.28(830), \mathrm{P}<0.001)$. However, the predictors included in the model accounted for only $5.8 \%$ of the variation in ATP-30 scores. According to it, those students had a more positive attitude toward psychiatry who identified as female, older and having any sort of exposure toward psychiatric specialty, direct involvement in psychiatric patient care, and reporting personal experience of mental illnesses.

Conclusion. Our study showed that medical students had a positive attitude towards psychiatry but female medical students, students with previous exposure to psychiatry, and students with longer psychiatry rotations tend to view psychiatry more positively. The generally positive trend towards psychiatry in Pakistan indicates the need to sustain improvements through 